This item was submitted to Loughborough's Research Repository by the author.

Items in Figshare are protected by copyright, with all rights reserved, unless otherwise indicated.

\title{
Unconventional pairing symmetry of layered superconductors caused by acoustic phonons
}

PLEASE CITE THE PUBLISHED VERSION

PUBLISHER

(C) American Physical Society

\section{VERSION}

VoR (Version of Record)

LICENCE

CC BY-NC-ND 4.0

\section{REPOSITORY RECORD}

Alexandrov, A.S.. 2019. "Unconventional Pairing Symmetry of Layered Superconductors Caused by Acoustic Phonons". figshare. https://hdl.handle.net/2134/4251. 
This item was submitted to Loughborough's Institutional Repository (https://dspace.lboro.ac.uk/) by the author and is made available under the following Creative Commons Licence conditions.

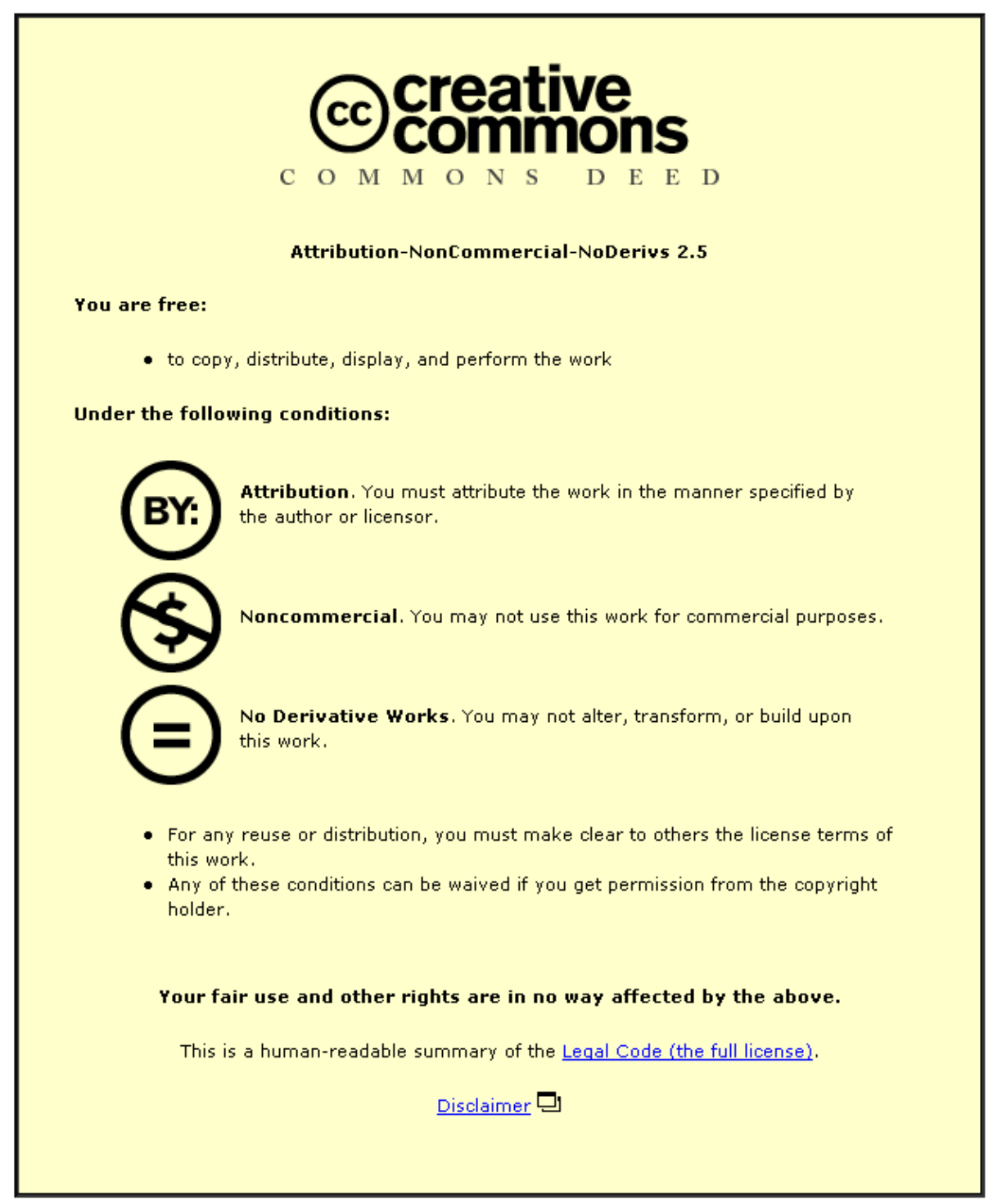

For the full text of this licence, please go to: http://creativecommons.org/licenses/by-nc-nd/2.5/ 


\title{
Unconventional pairing symmetry of layered superconductors caused by acoustic phonons
}

\author{
A. S. Alexandrov \\ Department of Physics, Loughborough University, Loughborough LE11 3TU, United Kingdom
}

(Received 2 November 2007; published 5 March 2008)

\begin{abstract}
An inevitable anisotropy of sound velocity in crystals makes the phonon-mediated attraction of electrons nonlocal in space providing unconventional Cooper pairs with a nonzero orbital momentum. As a result of this anisotropy, quasi-two-dimensional charge carriers weakly coupled with acoustic phonons undergo a quantum phase transition from a conventional $s$-wave to an unconventional $d$-wave superconducting state with less carriers per unit cell. In the opposite strong-coupling regime, rotational symmetry breaking appears as a result of a reduced Coulomb repulsion between unconventional bipolarons dismissing thereby some constraints on unconventional pairing in the Bose-Einstein condensation limit. The conventional acoustic phonons, and not superexchange, are shown to be responsible for the $d$-wave symmetry of cuprate superconductors, where the on-site Coulomb repulsion is large.
\end{abstract}

DOI: 10.1103/PhysRevB.77.094502

PACS number(s): 74.20.-z, 74.25.Bt, 74.40.+k, 74.72.- $\mathrm{h}$

A great number of observations, in particular, phasesensitive experiments, ${ }^{1}$ point to the unconventional $d$-wave symmetry of cuprate and some other superconductors (for a review, see Ref. 2). It has been thought for a long while that Cooper pairs in the Bardeen-Cooper-Schrieffer (BCS) theory with the conventional electron-phonon interaction (EPI) are singlets and their wave function is isotropic ( $s$ wave). ${ }^{3}$ This interaction has been thought to be local in space, so it could not lead to a higher angular-momentum pairing. Thus, it has gone unquestioned that the unconventional pairing requires unconventional electron-phonon interactions with specific optical phonons and poor screening, ${ }^{4-8}$ sometimes combined with antiferromagnetic fluctuations ${ }^{9}$ and vertex corrections, ${ }^{10}$ or nonphononic mechanisms of pairing (e.g., superexchange ${ }^{11}$ ), and a specific shape of the Fermi surface.

The pairing symmetry breaking is a many-body effect in accordance with a well-known quantum mechanics theorem, ${ }^{12}$ which states that the coordinate wave function of two particles does not become zero (or has no nodes) in the ground state. Hence, any superconductor should seem to be $s$ wave in the strong-coupling limit, ${ }^{13}$ where pairs are individual (e.g., bipolarons ${ }^{14}$ ) rather than overlapping Cooper pairs.

Here, the symmetry of the superconducting state mediated by conventional acoustic phonons is revised. The soundspeed anisotropy leads to a double surprise: (a) the BCS state of layered crystals is $d$ wave in a wide range of carrier densities; (b) the strong-coupling BEC state can break the rotational symmetry as well. The anisotropic EPI with acoustic phonons is proposed as the origin of the unconventional pairing owing to a giant sound-speed anisotropy in layered cuprate superconductors.

In the framework of the BCS theory, the symmetry of the order parameter $\Delta(\mathbf{k})$ and the critical temperature $T_{c}$ are found by solving the linearized "master" equation, ${ }^{3}$

$$
\Delta(\mathbf{k})=-\sum_{\mathbf{k}^{\prime}} V\left(\mathbf{k}, \mathbf{k}^{\prime}\right) \frac{\Delta\left(\mathbf{k}^{\prime}\right)}{2 \xi_{\mathbf{k}^{\prime}}} \tanh \left(\frac{\xi_{\mathbf{k}^{\prime}}}{2 k_{B} T_{c}}\right) .
$$

The interaction $V\left(\mathbf{k}, \mathbf{k}^{\prime}\right)$ comprises the attraction $-V_{p h}(\mathbf{q})$, mediated by phonons, and the Coulomb repulsion $V_{c}(\mathbf{q})$ as,

$$
\begin{aligned}
V\left(\mathbf{k}, \mathbf{k}^{\prime}\right)= & -V_{p h}(\mathbf{q}) \Theta\left(\omega_{D}-\left|\xi_{\mathbf{k}}\right|\right) \Theta\left(\omega_{D}-\left|\xi_{\mathbf{k}^{\prime}}\right|\right) \\
& +V_{c}(\mathbf{q}) \Theta\left(\omega_{p}-\left|\xi_{\mathbf{k}}\right|\right) \Theta\left(\omega_{p}-\left|\xi_{\mathbf{k}^{\prime}}\right|\right),
\end{aligned}
$$

where $V_{p h}(\mathbf{q})=C^{2} / N M c_{l}^{2}$ is the square of the matrix element of the electron-phonon interaction, ${ }^{15}$ divided by the square of the acoustic-phonon frequency, $\omega_{\mathrm{q}}=c_{l} q, c_{l}$ is sound velocity, $M$ is the ion mass, $N$ is the number of unit cells in the crystal, and $\xi_{\mathbf{k}}$ is the electron energy relative to the Fermi energy. The deformation potential matrix element $C$ is nearly $q$ independent near the $\Gamma$ point of the Brillouin zone in conventional metals ${ }^{15}$ and near extremum points of valence and conduction bands in doped semiconductors. ${ }^{16}$ While the validity of this approximation for cuprate superconductors has never been discussed, it affects none of our qualitative conclusions. The magnitude of $C$ is roughly the electron bandwidth in rigid metallic ${ }^{15}$ or semiconducting ${ }^{16}$ lattices. The electron momentum transfer $\mathbf{q}=\mathbf{k}-\mathbf{k}^{\prime}$ or its in-plane component has the magnitude $q=2^{1 / 2} k_{F}[1-\cos \psi]^{1 / 2}$ for the spherical or cylindrical Fermi surface, respectively, where $\psi$ is the angle between $\mathbf{k}$ and $\mathbf{k}^{\prime}$ and $\hbar k_{F}$ is the Fermi momentum. Theta functions in Eq. (2) $[\Theta(x)=1$ for positive $x$ and zero otherwise] account for a difference in frequency scales of the electron-phonon interaction $\omega_{D}$ and the Coulomb repulsion $\omega_{p} \gg \omega_{D}$, where $\omega_{D}$ and $\omega_{p}$ are the maximum phonon and plasmon energies, respectively.

If one neglects anisotropic effects, ${ }^{3}$ replacing $V_{p h}(\mathbf{q})$ and $V_{c}(\mathbf{q})$ by their Fermi-surface averages, $\quad V_{p h}(\mathbf{q}) \Rightarrow V_{p h}$, $V_{c}(\mathbf{q}) \Rightarrow V_{c}$, then there is only an $s$-wave solution of Eq. (1), $\Delta_{s}$, independent of $\mathbf{k}$. The sound-speed anisotropy actually changes the symmetry of the BCS state. While $c_{l}$ is a constant in the isotropic medium, it depends on the direction of $\mathbf{q}$ in any crystal. The anisotropy is particulary large in layered crystals such as cuprate superconductors, where an elastic stiffness constant in the $a-b$ plane is substantially greater than in the $c$ direction (see Refs. 17 and 18 and references therein). As an example, the measured velocity of longitudinal ultrasonic waves along $a-b$ plane, $c_{\|}=4370 \mathrm{~ms}^{-1}$, is almost twice larger than that along $c$ axis, $c_{\perp}=2670 \mathrm{~ms}^{-1}$, in $\mathrm{Bi}_{2} \mathrm{Sr}_{2} \mathrm{CaCu}_{2} \mathrm{O}_{8+y} .{ }^{18}$ It makes $V_{p h}(\mathbf{q})$ anisotropic, 


$$
V_{p h}(\mathbf{q})=\frac{C^{2}}{N M c_{\perp}^{2}\left(1+\alpha q_{\|}^{2} / q^{2}\right)},
$$

where $\alpha=\left(c_{\|}^{2}-c_{\perp}^{2}\right) / c_{\perp}^{2}$ is the anisotropy coefficient, which is about 2 in cuprates. Also, the Coulomb repulsion is $q$ dependent, $V_{c}(\mathbf{q})=4 \pi e^{2} / V \epsilon_{0}\left(q^{2}+q_{s}^{2}\right)$. In the framework of the random phase approximation, the inverse screening radius squared is found as $q_{s}^{2}=8 \pi e^{2} N(0) / V \epsilon_{0}$, with the density of states (per spin) $N(0)$ at the Fermi surface. Here, $\epsilon_{0}$ is the (in-plane) static dielectric constant of the host cuprate lattice of the volume $V$.

Solving the master equation [Eq. (1)] with twodimensional (2D) electron spectrum, one can expand $\Delta(\mathbf{k})$ $=\Sigma_{m} \Delta_{m} \exp (i m \phi) \quad$ and $V_{p h, c}(\mathbf{q})=\Sigma_{m} V_{p h, c}\left(q_{\perp}, m\right) \exp [i m(\phi$ $\left.\left.-\phi^{\prime}\right)\right]$ in series of the eigenfunctions of the $c$-axis component of the orbital angular momentum, where $\phi$ and $\phi^{\prime}$ are polar angles of the in-plane momenta, $\mathbf{k}_{\|}$and $\mathbf{k}_{\|}^{\prime}$, respectively.

The solution for the $m$ component of the order parameter $(m=0, \pm 1, \pm 2, \ldots)$ is found in the form $\Delta_{m}=\Delta_{m}^{(1)} \Theta\left(\omega_{D}-\left|\xi_{\mathbf{k}}\right|\right)+\Delta_{m}^{(2)} \Theta\left(\omega_{p}-\left|\xi_{\mathbf{k}}\right|\right) \Theta\left(\left|\xi_{\mathbf{k}}\right|-\omega_{D}\right)$ with different values of $\Delta_{m}^{(1)}$ and $\Delta_{m}^{(2)}$ below and above the cutoff energy $\omega_{D}$, respectively. Integrating in Eq. (1) over $\xi_{\mathbf{k}^{\prime}}, \quad \phi^{\prime}$ (using the integral $\int_{0}^{2 \pi} d \psi \cos (m \psi) /(1-p \cos \psi)$ $\left.=2 \pi\left[1-\left(1-p^{2}\right)^{1 / 2}\right]^{m} / p^{m}\left(1-p^{2}\right)^{1 / 2}\right)$, and finally over $q_{\perp}$ yields the following pair of equations:

$$
\begin{gathered}
\Delta_{m}^{(1)}\left[1-\left(\lambda_{m}-\mu_{m}\right) \ln \frac{1.14 \omega_{D}}{k_{B} T_{c}}\right]+\Delta_{m}^{(2)} \mu_{m} \ln \frac{\omega_{p}}{\omega_{D}}=0, \\
\Delta_{m}^{(2)}\left[1+\mu_{m} \ln \frac{\omega_{p}}{\omega_{D}}\right]+\Delta_{m}^{(1)} \mu_{m} \ln \frac{1.14 \omega_{D}}{k_{B} T_{c}}=0 .
\end{gathered}
$$

Here, $\lambda_{m}$ and $\mu_{m}$ are the phonon-mediated attraction and the Coulomb pseudopotential in the $m$-pairing channel, given, respectively, by

$$
\frac{\lambda_{m}}{\lambda}=\delta_{m, 0}+\frac{\alpha}{2 \sqrt{\gamma}} \int_{0}^{\gamma} \frac{d x[x+1-\sqrt{x(x+2)}]^{m}}{\sqrt{x+2}}
$$

and

$$
\frac{\mu_{m}}{\mu_{c}}=\frac{\sqrt{\tilde{\gamma}}}{2} \int_{0}^{\tilde{\gamma}} \frac{d x[x+\beta+1-\sqrt{(x+\beta)(x+\beta+2)}]^{m}}{\sqrt{x(x+\beta)(x+\beta+2)}},
$$

where $\lambda=N(0) C^{2} / N M c_{\|}^{2}, \gamma=\pi^{2} / 2 d^{2} k_{F}^{2}(1+\alpha), d$ is the interlayer distance, $\tilde{\gamma}=\gamma(1+\alpha), \quad \mu_{c}=4 e^{2} d^{2} N(0) / \pi V \epsilon_{0}$, and $\beta$ $=q_{s}^{2} / 2 k_{F}^{2}$ [note that $\lambda, \mu_{c}$, and $q_{s}$ do not depend on the carrier density since $N(0)$ is roughly constant in the quasi-twodimensional Fermi gas].

The effective attraction of two electrons in the Cooper pair with nonzero orbital momentum turns out finite at any finite anisotropy, $\alpha \neq 0$, but numerically smaller than in the $s$-channel [Fig. 1 (inset)], as is also seen from its analytical expressions for $s$-wave pairing, $m=0\left(\lambda_{s}\right), p$-wave pairing, $m=1\left(\lambda_{p}\right)$, and for $d$-wave pairing, $m=2\left(\lambda_{d}\right)$, obtained by integrating in Eq. (6). When the interlayer distance is much larger than the wavelength of electrons, $\gamma \ll 1$, one obtains

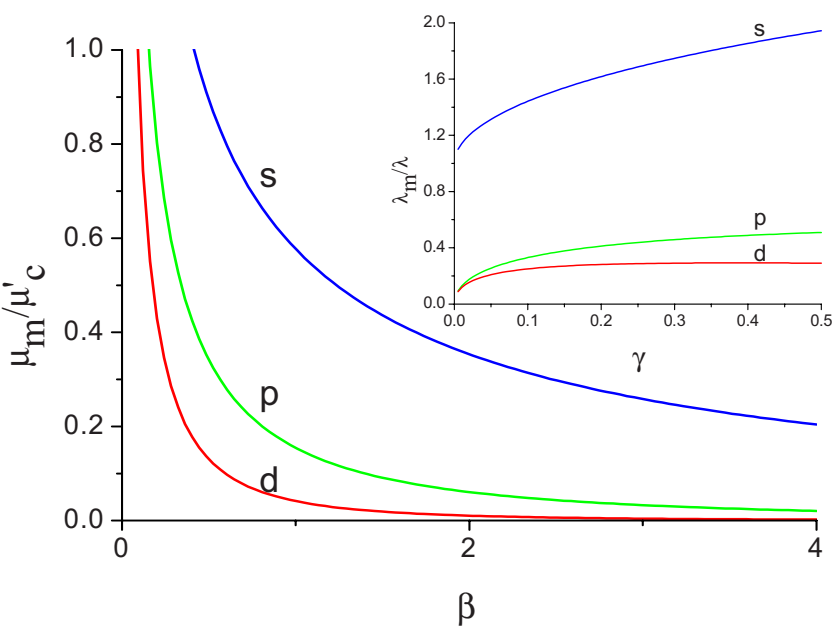

FIG. 1. (Color online) The Coulomb repulsion $\mu_{m}$ as a function of the ratio of the electron wavelength to the screening length squared $\left(\beta=q_{S}^{2} / 2 k_{F}^{2}\right)$, and the electron-phonon coupling constant $\lambda_{m}$ as a function of the ratio of the electron wavelength to the interplane distance squared, $\gamma=\pi^{2} / 2 d^{2} k_{F}^{2}(1+\alpha)$ for $\alpha=4$ (inset) in $s, p$, and $d$ pairing channels. Here, $\mu_{c}^{\prime}=\mu_{c} \tilde{\gamma}$.

$\lambda_{s} \approx \lambda, \lambda_{p} \approx \lambda \alpha(\gamma / 2)^{1 / 2} / 2$, and $\lambda_{d} \approx \lambda_{p}$. In the opposite limit, $\gamma \gg 1$, one finds $\lambda_{s} \approx \lambda(1+\alpha), \quad \lambda_{p} \approx \lambda \alpha(2 / \gamma)^{1 / 2} / 3$, and $\lambda_{d} \approx \lambda_{p} / 5$.

The Coulomb repulsion is much smaller in the unconventional pairing states than in the conventional $s$-wave state (Fig. 1), which is also seen from the analytical expression for $\mu_{m}$ [Eq. (7)]. If $\tilde{\gamma} \leq \beta$, the repulsion constant $\mu_{m}$ drops as $1 / \beta^{m+1}$ in the $m$ channel at strong screening, when $\beta \gg 1$. It provides a wide region with unconventional pairs in the " $\gamma-\beta$ " parameter space, in spite of the lower values of their electron-phonon coupling constants (Fig. 2). Indeed, the critical temperature for $m$-Cooper pairing is

$$
T_{c m}=1.14 \omega_{D} \exp \left[-1 /\left(\lambda_{m}-\mu_{m}^{*}\right)\right],
$$

where $\mu_{m}^{*}=\mu_{m} /\left[1+\mu_{m} \ln \left(\omega_{p} / \omega_{D}\right)\right]$, as found from Eqs. (4) and (5). For a fixed set of material parameters (which define $\lambda$ and $\mu_{c}$ ), the physically realized superconducting instability appears in the angular-momentum channel with the highest $T_{c m}$. A minimum (i.e., critical) ratio $\lambda / \mu_{c}$ for the existence of superconductivity in the $m$ channel is determined by the condition $\lambda_{m}=\mu_{m}^{*}$ as the function of the parameters $\gamma$ and $\beta$. Naturally, the $m$-pairing state with the lowest value of the critical ratio has the highest $T_{c}$. The critical $d$-wave surface, $\lambda / \mu_{c}=S(\gamma, \beta)$, defined using $\lambda_{m}=\mu_{m}^{*}$, is found below $s$-wave and $p$-wave surfaces, if $\beta \gtrsim 1$, so that the $d$-wave state is physically realized in this region of parameters, as seen from Fig. 2.

Higher-momentum states, $m \geqslant 3$, have even a smaller Coulomb repulsion at large $\beta$ [Eq. (7)], so that they can be realized as well, if $\gamma$ is so small, that $\lambda_{m}$ in Eq. (6) is almost $m$ independent for $m \geqslant 1$. On the other hand, an in-plane anisotropy of the sound velocity, compatible with the symmetry of the perovskite lattice, makes $d$-wave state more stable compared with the higher-momentum states. Naturally, if the sound speed is enhanced along the diagonal di- 

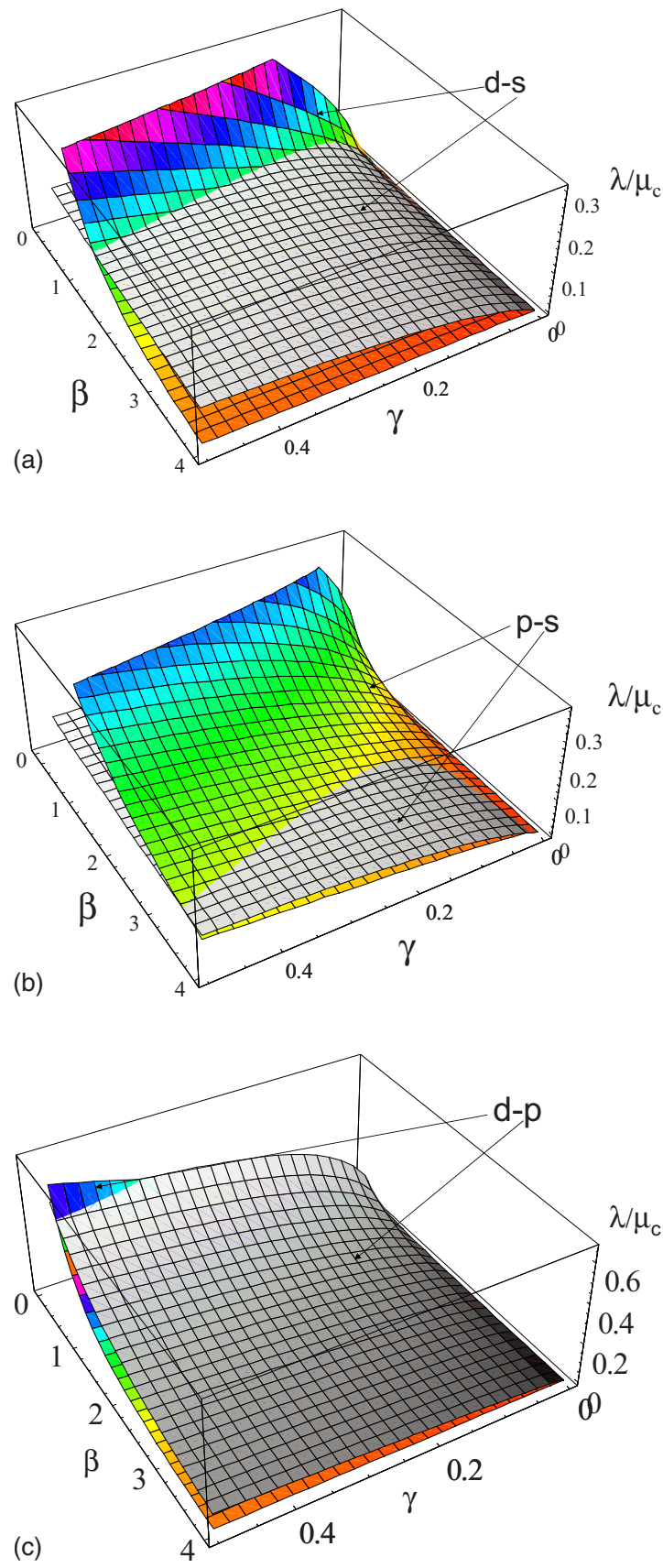

FIG. 2. (Color online) The critical values of the electron-phonon coupling $\lambda$ for $d$-wave (bright colors) versus $s$-wave (gray color) (d-s), $p$-wave (bright colors) versus $s$-wave (gray color) (p-s), and $d$-wave (bright colors) versus $p$-wave (gray color) Cooper pairs, as functions of the parameters $\beta$ and $\gamma$. The Tolmachev-MorelAnderson logarithm is set here at $\mu_{c} \ln \left(\omega_{p} / \omega_{D}\right)=3$ and $\alpha=3$.

rections of the in-plane primitive cell, the $d$-wave order parameter would be zero along diagonals of the $2 \mathrm{D}$ Brillouin zone, where it changes its sign.

Using the simplest parabolic approximation for a 2Delectron energy spectrum, we can draw some conclusions on the carrier-density evolution of the order-parameter symmetry. Within this approximation, $k_{F}^{2}=2 \pi d n$ and $N(0)$ $=m V / 2 \pi d \hbar^{2}$, where $n=2 x / \Omega$ is the carrier density and $x$ is the doping level as in $\mathrm{La}_{2-x} \mathrm{Sr}_{x} \mathrm{CuO}_{4}$ with the unit cell vol-

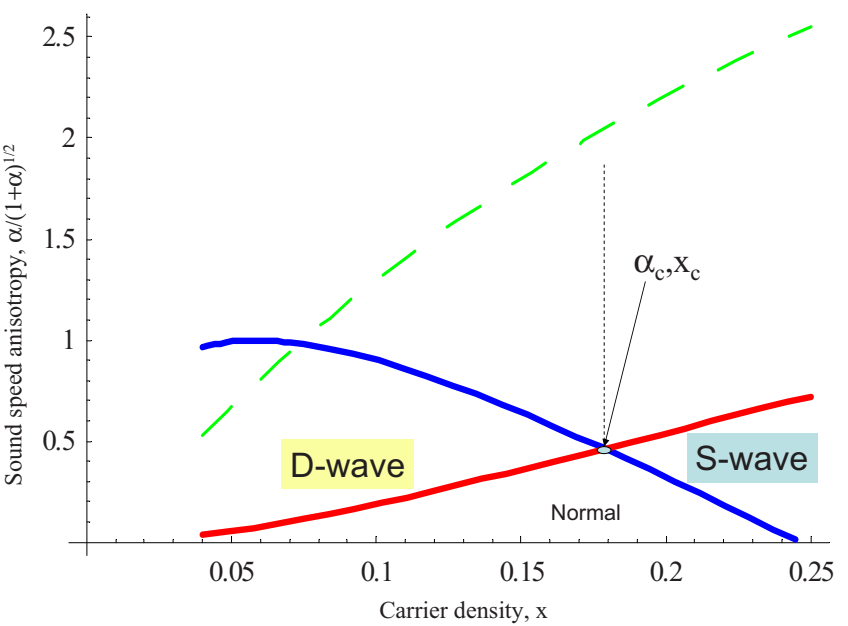

FIG. 3. (Color online) Critical sound-speed anisotropy, $\alpha /(1+\alpha)^{1 / 2}=\left(c_{\|}^{2}-c_{\perp}^{2}\right) / c_{\|} c_{\perp}$, as a function of doping, $x$, for $\lambda=\mu_{c} / 12$ (solid lines correspond to $d$ and $s$ states, and dashed line to $p$ state). With increasing carrier density, there is a quantum phase transition at $x=x_{c}$ from a $d$-wave to an $s$-wave superconductor, when $\alpha>\alpha_{c}$, and two quantum phase transitions from $d$-wave to the normal state and from the normal state to the $s$-wave state when $\alpha<\alpha_{c}$.

ume $\Omega$. The ratio of the parameters $\beta=m e^{2} \Omega / 2 \pi \hbar^{2} d^{2} \epsilon_{0} x$ and $\tilde{\gamma}=\pi \Omega / 8 d^{3} x \approx 0.044 / x$ is independent of the carrier density, $\beta / \tilde{\gamma}=4 m e^{2} d / \pi^{2} \hbar^{2} \epsilon_{0}$, which is approximately 5 for the values of $m=4 m_{e}$ and $\epsilon_{0}=10$. Fixing the value of the EPI constant at $\lambda=\mu_{c} / 12$ (which corresponds to the weak-coupling BCS regime with $\lambda \approx 0.1$ since $\mu_{c}$ is of the order of 1 ) and taking $\mu_{c} \ln \left(\omega_{p} / \omega_{D}\right)=3$, we draw the anisotropy-doping phase diagram (Fig. 3), with the critical lines for $s, p$, and $d$ order parameters, defined by $\lambda_{m}=\mu_{m}^{*}$. The state with the lowest magnitude of the anisotropy, $\alpha /(1+\alpha)^{1 / 2}$, is physically realized since it has the highest $T_{c}$. At substantial doping, the screening length becomes larger than the typical wavelength of electrons, $\beta \rightarrow 0$, so that the $s$-wave state is the ground state at a large number of carriers per unit cell for any anisotropy. On the contrary, the Coulomb repulsion is reduced to the local interaction at a low doping, $\beta \rightarrow \infty$, and $d$-wave Cooper pairs are the ground state even at very low value of the anisotropy (Fig. 3). Interestingly, $s$ and $d$ states turn out degenerate at some intermediate value of doping, $x=x_{c}$. Hence, there is a quantum phase transition with increasing doping from $d$ - to $s$-superconducting state, if $\alpha>\alpha_{c}$, and from $d$ to the normal state and then to the $s$-wave superconductor, if $\alpha<\alpha_{c}$ (see Fig. 3).

In the strong-coupling regime, $\lambda \gtrsim 1$, the pairing is individual, ${ }^{14}$ in contrast with the collective Cooper pairs. While the Bose condensate of individual bipolarons could break the symmetry on a discreet lattice, ${ }^{19,20}$ here I propose a symmetry breaking mechanism, which works even in a continuum model, where the ground state, it would seem, be s wave $^{13}$ to satisfy the theorem. ${ }^{12}$

The unscreened Fröhlich EPI with optical phonons in layered ionic lattices such as cuprates has been suggested by us as the key for pairing. ${ }^{14}$ Acting alone it cannot overcome the direct Coulomb repulsion, but almost nullifies it since $\epsilon_{0} \gg 1$. 
That allows the weaker deformation potential [Eq. (3)] to bind carriers into real-space bipolarons, if $\lambda \geqslant 0.5 .{ }^{14}$ The corresponding potential, $V(\mathbf{r})=-\Sigma_{\mathbf{q}} V_{p h}(\mathbf{q}) \exp (i \mathbf{q} \cdot r)$, is nonlocal in real space,

$$
V(\mathbf{r})=-V_{p h} \Omega\left[\frac{\delta(\mathbf{r})}{d}+\frac{\alpha}{4 \pi(1+\alpha)^{1 / 2} r^{3}}\right],
$$

falling as $1 / r^{3}$ at the distance $r \gg d$ between two carriers in the plane, where $V_{p h}=C^{2} / M c_{\perp}^{2}$. While its local part $[\propto \delta(\mathbf{r})=\delta(x) \delta(y)]$ is negated by the strong on-site repulsion $U$, the second nonlocal part provides bound pairs of different symmetries with the binding energies $\Delta_{s}>\Delta_{p}>\Delta_{d}>\cdots$, in agreement with the theorem.

However, there is the residual Coulomb repulsion between bipolarons $v_{c}(R)$, significantly reduced by optical phonons. If we approximate the bipolaron as a point charge $2 e$, then $v_{c}(R) \approx 4 e^{2} / \epsilon_{0} R$. Since bipolarons have a finite extension $\xi$, there are corrections to the Coulomb law. The bipolaron has no dipole moment, hence the most important correction at large distances between two bipolarons, $R \gg \xi$, comes from the charge-quadrupole interaction, ${ }^{12}$ $v_{c}(R)=4 e^{2}\left(1 \pm \eta \xi^{2} / R^{2}\right) / \epsilon_{0} R$, where $\eta$ is a number of the order of 1 , and plus and/or minus signs correspond to bipolarons in the same or different planes, respectively. The dielectric screening, $\epsilon_{0}$ is highly anisotropic in cuprates, where the in-plane dielectric constant $\epsilon_{0 \|}$ is much larger than the out-of-plane one $\epsilon_{0 \perp} \cdot{ }^{21}$ Hence, the interplane repulsion provides the major contribution to the condensation energy. Since $\xi^{2} \propto 1 / \Delta$, the repulsion of unconventional bipolarons with smaller binding energies, $\Delta_{d}, \Delta_{p}<\Delta_{s}$, is reduced compared with the repulsion of $s$-wave bipolarons. As a result, with increasing carrier density, we anticipate a transition from BEC of $s$-wave bipolarons to BEC of more extended $p$ and $d$-wave real-space pairs in the strong-coupling limit.

Several authors ${ }^{11}$ have remarked that superexchange, and not phonons, is responsible for the symmetry breaking in unconventional superconductors such as doped cuprates. Here, I arrive at the opposite conclusion. Indeed, superexchange interaction $J$ is proportional to the electron hopping integral $t^{2}$ divided by the on-site Coulomb repulsion (Hubbard $U), J=4 t^{2} / U$, estimated as $J \approx 0.15 \mathrm{eV}$ in cuprates. ${ }^{11}$ This should be compared with the acoustic-phonon pairing interaction $V_{p h}$, which is roughly the Fermi energy, $V_{p h} \approx E_{F} \approx 4 t$ in a metal ${ }^{15}$ or the bandwidth squared divided by the ion-ion interaction energy of the order of the nearestneighbor Coulomb repulsion, $M c_{l}^{2} \approx V_{c}$ in a doped insulator. $^{16}$ The small ratio of two interactions, $J / V_{p h}$ $\approx t / U \ll 1$ or $J / V_{p h} \approx V_{c} / U \ll 1$, and the giant sound-speed anisotropy ${ }^{17,18}$ favor conventional EPI as the origin of the unconventional pairing both in underdoped cuprates, where the pairing is individual, ${ }^{14}$ and in overdoped samples apparently with Cooper pairs. ${ }^{3}$

Nowadays, compelling evidence for a strong EPI has arrived from isotope effects, ${ }^{22}$ more recent high resolution angle resolved photoemission spectroscopies, ${ }^{23}$ and a number of earlier optical, ${ }^{24-27}$ neutron-scattering, ${ }^{28}$ and recent inelastic scattering measurements ${ }^{29}$ in cuprates. Whereas calculations based on the local spin-density approximation (LSDA) often predict negligible EPI, the inclusion of Hubbard $U$ in the LSDA $+U$ calculations greatly enhances its strength. ${ }^{30}$ While the coupling with particular phonon modes is quite different, ${ }^{23,29,30}$ EPI with conventional acoustic phonons and the substantial sound-speed anisotropy explain alone the unconventional symmetry of cuprate superconductors.

I thank A. F. Andreev, I. Bozovic, J. P. Hague, V. V. Kabanov, P. E. Kornilovitch, K. I. Kugel, M. L. Kulic, I. I. Mazin, J. H. Samson, and L. M. Satarov for valuable discussions. The work was supported by EPSRC (UK) (Grants No. EP/C518365 and No. EP/D035589).
${ }^{1}$ C. C. Tsuei and J. R. Kirtley, Rev. Mod. Phys. 72, 969 (2000).

${ }^{2}$ J. Annett, N. Goldenfeld, and A. J. Legget, J. Low Temp. Phys. 105, 473 (1996).

${ }^{3}$ J. Bardeen, L. N. Cooper, and J. R. Schrieffer, Phys. Rev. 108, 1175 (1957).

${ }^{4}$ A. A. Abrikosov, Phys. Rev. B 52, R15738 (1995).

${ }^{5}$ V. Hizhnyakov and E. Sigmund, Phys. Rev. B 53, 5163 (1996).

${ }^{6}$ Z. X. Shen, A. Lanzara, S. Ishihara, and N. Nagaosa, Philos. Mag. B 82, 1349 (2002).

${ }^{7}$ M. L. Kulic, J. Supercond. Novel Magn. 19, 213 (2006).

${ }^{8}$ F. D. Klironomos and S. W. Tsai, Phys. Rev. B 74, 205109 (2006).

${ }^{9}$ I. Schnell, I. I. Mazin, and A. Y. Liu, Phys. Rev. B 74, 184503 (2006).

${ }^{10}$ J. P. Hague, Phys. Rev. B 73, 060503(R) (2006).

${ }^{11}$ P. W. Anderson, P. A. Lee, M. Randeria, T. M. Rice, N. Tiverdi, and F. C. Zhang, J. Phys.: Condens. Matter 16, R755 (2004).

${ }^{12}$ L. D. Landau and E. M. Lifshitz, Quantum Mechanics (Pergamon, Oxford, 1977) pp. 59\&237.
${ }^{13}$ J. Quintanilla, B. L. Gyorffy, J. F. Annett, and J. P. Wallington, Phys. Rev. B 66, 214526 (2002).

${ }^{14}$ A. S. Alexandrov, Theory of Superconductivity: From Weak to Strong Coupling (Taylor and Francis, London, 2003).

${ }^{15}$ J. Bardeen and D. Pines, Phys. Rev. 99, 1140 (1955).

${ }^{16}$ A. Anselm, Introduction of Semiconductor Theory (Prentice and Hall, New Jersey, 1981).

${ }^{17}$ A. Migliori, W. M. Visscher, S. Wong, S. E. Brown, I. Tanaka, H. Kojima, and P. B. Allen, Phys. Rev. Lett. 64, 2458 (1990).

${ }^{18}$ F. Chang, P. J. Ford, G. A. Saunders, L. Jiaqiang, D. P. Almond, B. Chapman, M. Cankurtaran, R. B. Poeppel, and K. C. Goretta, Supercond. Sci. Technol. 6, 484 (1993).

${ }^{19}$ A. S. Alexandrov, Int. J. Mod. Phys. B 14, 3298 (2000).

${ }^{20}$ A. F. Andreev, Pis'ma Zh. Eksp. Teor. Fiz. 79, 100 (2004).

${ }^{21}$ Z. Zhai, P. V. Parimi, J. B. Sokoloff, S. Sridhar, and A. Erb, Phys. Rev. B 63, 092508 (2001).

${ }^{22}$ G. M. Zhao and D. E. Morris, Phys. Rev. B 51, 16487 (1995); G.-M. Zhao, M. B. Hunt, H. Keller, and K. A. Müller, Nature (London) 385, 236 (1997); R. Khasanov, D. G. Eshchenko, H. 
Luetkens, E. Morenzoni, T. Prokscha, A. Suter, N. Garifianov, M. Mali, J. Roos, K. Conder, and H. Keller, Phys. Rev. Lett. 92, 057602 (2004).

${ }^{23}$ A. Lanzara, P. V. Bogdanov, X. J. Zhou, S. A. Kellar, D. L. Feng, E. D. Lu, T. Yoshida, H. Eisaki, A. Fujimori, K. Kishio, J. I. Shimoyana, T. Noda, S. Uchida, Z. Hussain, and Z. X. Shen, Nature (London) 412, 510 (2001); G.-H. Gweon, T. Sasagawa, S. Y. Zhou, J. Craf, H. Takagi, D.-H. Lee, and A. Lanzara, ibid. 430, 187 (2004); X. J. Zhou, J. Shi, T. Yoshida, T. Cuk, W. L. Yang, V. Brouet, J. Nakamura, N. Mannella, S. Komiya, Y. Ando, F. Zhou, W. X. Ti, J. W. Xiong, Z. X. Zhao, T. Sasagawa, T. Kakeshita, H. Eisaki, S. Uchida, A. Fujimori, Z.-Y. Zhang, E. W. Plummer, R. B. Laughlin, Z. Hussain, and Z.-X. Shen, Phys. Rev. Lett. 95, 117001 (2005).

${ }^{24}$ D. Mihailovic, C. M. Foster, K. Voss, and A. J. Heeger, Phys. Rev. B 42, 7989 (1990).

${ }^{25}$ P. Calvani, M. Capizzi, S. Lupi, P. Maselli, A. Paolone, P. Roy, S.
W. Cheong, W. Sadowski, and E. Walker, Solid State Commun. 91, 113 (1994).

${ }^{26}$ R. Zamboni, G. Ruani, A. J. Pal, and C. Taliani, Solid State Commun. 70, 813 (1989).

${ }^{27}$ T. Timusk, C. C. Homes, and W. Reichardt, in Anharmonic Properties of High Tc Cuprates, edited by D. Mihailovic, G. Ruani, E. Kaldis, and K. A. Müller (World Scientific, Singapore, 1995), p. 171.

${ }^{28}$ T. R. Sendyka, W. Dmowski, T. Egami, N. Seiji, H. Yamauchi, and S. Tanaka, Phys. Rev. B 51, 6747 (1995); T. Egami, J. Low Temp. Phys. 105, 791 (1996).

${ }^{29}$ D. Reznik, L. Pintschovius, M. Ito, S. Iikubo, M. Sato, H. Goka, M. Fujita, K. Yamada, G. D. Gu, and J. M. Tranquada, Nature (London) 440, 1170 (2006).

${ }^{30}$ P. Zhang, S. G. Louie, and M. L. Cohen, Phys. Rev. Lett. 98, 067005 (2007). 